\begin{tabular}{ll} 
Nucleic acids come clean & 856 \\
\hline Special dispensation & 859 \\
\hline Welcome to the machine & 862 \\
\hline Box 1: From start to finish & 856 \\
\hline Box 2: A chip off the old 'bot & 859 \\
\hline
\end{tabular}

\title{
Automatic for the people
}

More and more scientists now see advantages in automating some of their more repetitive or error-prone tasks. Michael Eisenstein takes a look at systems that are helping to bring robotics into the academic and clinical research laboratory.

Robots-they are not just for big pharma anymore. To be sure, the extent of automation in the academic and clinical sector may not be at the room-filling scale seen in the worlds of drug development and industrial production, but as biological screens swell in size, the incentive to automate repetitive tasks becomes more compelling. "We have a research program centered on the functional interrogation of cDNAs that has a high dependence on automation," says Brian Seed, a researcher who also comanages a shared genomics automation core at Massachusetts General Hospital, "and we try to encourage people to rely on our automation group instead of going it alone."

Wendy Lauber, global marketing manager at Tecan, sees this as a growing trend. "Our non-industry customers are discovering the benefits of automating parts of the process from which they would like to free up their people and reduce the manual labor," she says. "And most importantly, they're finding the need for consistency of the data that they're using." As the number of wells and plates in an experiment grows, the physical strain on the research staff-and the likelihood of a careless error-increases, and Seed emphasizes this benefit of automation over any potential speed or cost gains. "It provides you with greater overall reliability by diminishing human error," he says, "and that is the most important thing that it does."

Genetic research has been a key area of development in this regard, with its reliance on repetitive tasks (for example, DNA minipreps and PCR setup) and a broad range of increasingly high-throughput screens (for example, RNA interference studies and genetic interaction screens), and several companies are now offering

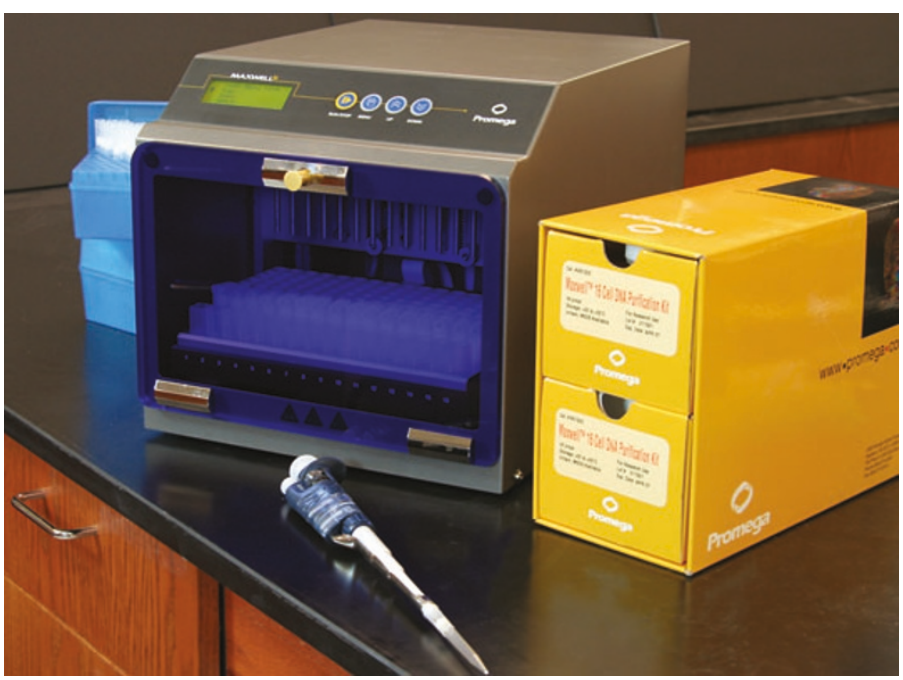

The Maxwell 16 System from Promega offers benchtop automation of nucleic-acid purification. (Courtesy of Promega.)

automated solutions that attempt to simplify these routines for laboratories working at virtually every level of throughput.

\section{Pick and choose}

Most genetic assays begin with the selection of positive clones-identifying cells that show a particular growth or resistance, or other gene expression phenotype, and then transferring them from the culture dish to a tube or multiwell plate for further analysis. As every technician knows, this can be a tedious exercise in eye strain, and for many of the large-scale screens currently being performed, manual picking is an unrealistic option.

Genetix manufactures a wide variety of clone-picking instruments and touts the claim that their instruments served as the standard platform for six out of seven sequencing centers involved in the Human
Genome Project. Available options range from the QPix2, a benchtop system suitable for picking clones, replicating plates or gridding clones onto membranes, to the MegaPix, a high-capacity workstation with an automated plate delivery system. These platforms digitally image each plate to identify clones of interest based on user-established parameters, and then use a 96 pin-based transfer system to mediate the transfer, with the pins being sterilized by washing, heat and ultraviolet light between picks. These systems are designed primarily for bacteria, yeast and viral plaques, but Genetix also offers the ClonePix instrument for the picking and transfer of adherent mammalian cell lines.

The PM-1s Colony Picker, manufactured by Microtec Nition but distributed by B-Bridge International in North 
America, may offer a more budget-friendly alternative for the smaller lab, with a lower price tag and a smaller benchtop footprint (roughly 350 square centimeters), although this comes at the cost of reduced throughput. The PM-1s uses a similar picking process, imaging plates with a built-in four-megapixel digital camera, then using a needle to pick colonies. The

PM-1s uses a three-step sterilization process-brushing, ethanol rinse and heatto clean its needles, but also features the option of automatic needle replacement between picks.

For labs that have already made the decision to automate with one of Hamilton Life Science Robotics' MICROLAB platforms, the EasyPick module is an option. EasyPick incorporates a light table, a charge-coupled device camera and software for the analysis of captured colony images. "You can take pictures and then do the selection of the right colonies that you want to pick," says Roland Borner, product manager for genomics, "so the user has different things that they can choose from, such as how big the colonies should be, or the shape or color of

\section{BOX 1 FROM START TO FINISH}

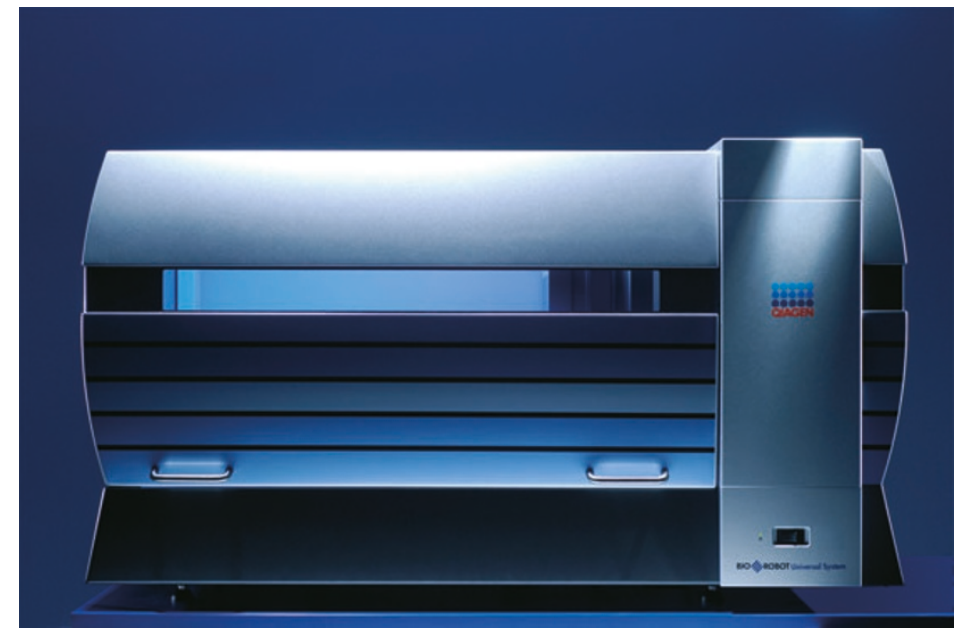

The BioRobot Universal System from Qiagen offers standardized solutions for a variety of nucleic-acid applications. (Courtesy of Qiagen.)

the colonies." The system is capable of automated isolation of yeast, bacteria and phage plaques, and based on the configuration of the individual MICROLAB system, users can set up programs that follow this process with other sample preparation functions, such as nucleicacid or protein purification.

\section{Nucleic acids come clean}

Collecting large numbers of clones-or tail clips or blood samples, for that matter-just leads the investigator to yet another highly repetitive, but potentially problematic, process: nucleic acid preparation. In an industry-scale setting, this will often be handled by adding just one
The manual transfer of PCR plates from an automated preparative instrument to an automated thermocycler may not be the most burdensome of tasks, but it does introduce the need for human participation-and an opportunity for human error-into an otherwise fully automated process, as well as raise the risk of contamination or biohazard exposure for those working with forensic or clinical samples.

Roche Diagnostics and Abbott Molecular are among a small number of companies helping users to avoid this speed bump by offering robotic workstations that integrate independent automated-sample preparation with amplification and detection units. Roche offers the COBAS family of platforms, which include the AmpliPrep Instrument, for the preparation of DNA or RNA from biological samples, and the COBAS TaqMan Analyzer, a highcapacity RT-PCR platform. Each can be used independently, but the two can also be configured for automated sample transfer between instruments. Abbott also previewed a similar instrument the $m 2000$, this past July, at the annual meeting of the American Association for Clinical Chemistry. Both instruments are designed for use in a molecular diagnostics setting, and feature systemspecific screens for a variety of pathogens, including HIV-1 and hepatitis $C$ virus.

Cepheid has taken a different approach with their GeneXpert system, a single instrument that operates as a fully integrated diagnostic RT-PCR platform, capable of going from raw sample to result in half an hour. According to Cepheid CEO John Bishop, the system is designed for both safety and efficiency. "You basically have a totally molded system that's all closed, [with] a rotating valve at the bottom," he explains. "Based on commands within the system, it rotates that valve around, and as the valve rotates it opens up various ports for reagents for various processing steps within the cartridge." GeneXpert can handle up to 16 samples simultaneously, each of which can be processed with a distinct preparation and amplification protocol.

A wide range of sample formats can be processed in this platform, including bodily fluids, solid matter and even aerosols. In fact, this technology saw an early test in the wake of 2001's anthrax incidents, when the US Postal Service contracted the development of a Biohazard Detection System that incorporates a GeneXpert module for rapid detection of airborne anthrax spores. According to Bishop, "the [US Postal Service] system has never given a false positive in over 3.2 million test results to date, while also maintaining a very high level of sensitivity." The system shows equally strong sensitivity and specificity in clinical settings, and recently received US Food and Drug Administration clearance to market a Group B Streptococcus diagnostic product, and a variety of other tests are now under development. Although the throughput may be lower than the other 96-well plate-based robotic systems, Bishop believes the simplicity of their systemultimately designed for use by nonresearch professionals-offers some compensation for this. "The system is showing a real ability to be user-friendly," he says. 
more robotic module, but for small- and medium-throughput labs, stand-alone solutions are a more economical and realistic option. "Scientists in the academic and clinical realm don't want to spend their time troubleshooting or optimizing systems," says Hemanth Shenoi, product manager for integrated systems at Promega. "They want to be able to get consistant results because what they're working on isn't integration or robotics, it's biology."

With this in mind, Promega developed the Maxwell 16, a benchtop instrument that uses a cartridge-based system to perform simultaneous genomic DNA or total RNA preparation for up to 16 raw samples. Each sealed cartridge contains prealiquoted buffers and paramagnetic particles functionalized for the purification process. Rather than adding and removing buffers from the sample well, the Maxwell 16 instrument uses an automated plunger to transfer the nucleic acid-bound particles from well to well. This plunger also provides crushing action at the lysis stage, allowing the use of many different sample materials. "I've used it with prokaryotic and eukaryotic cells," says Daniel Kephart, head of the Promega Scientific Applications Support Group. "You can actually put a $1-\mathrm{cm}$ piece of mouse tail right into the cartridge and there's enough force to smash that sample up with high efficiency and extract the nucleic acids." The system also benefits from a small footprint, about the size of a laptop computer, which makes it easy to fit into a laboratory in a standard hood, or even a mobile laboratory setting.

The MagNA Pure instruments from Roche Applied Science use a process that they call 'magtration'; purifying nucleic acids on magnetic particles within specially designed pipette tips. The MagNA Pure Compact instrument is designed for low-throughput applications, and allows the automated preparation of RNA or DNA from one to eight samples. The MagNA Pure LC offers greater throughput, handling up to 32 samples at once, but is also capable of following purification with the preparation of samples for standard or real-time PCR. "Having a PCR setup option within the instrument is a lower investment for the customer, compared to buying a separate workstation for setup," says Roche global marketing manager Isabel Schmitt. "Full automation [also] offers high reliability and reproducibility." Both systems also feature bar-code readers for sample tracking, and a simple software interface that allows users to easily configure parameters relating to sample type or, for the MagNA Pure LC unit, PCR format.

Qiagen's BioRobot platforms straddle the boundary separating smaller standalone systems from more open-ended workstations, but still rely heavily on the use of standardized solutions for each application. "We offer a broad range of Qiagen-validated applications in the area of sample preparation for these systems," says Leibinger. "It takes three days to set up one of our larger instruments in the lab, but then it's done and it's 
working, without further optimization steps." The simplest system, the BioRobot EZ-1, offers a low-throughput cartridgebased platform for preparing nucleic acids from one to six samples, but Qiagen also offers larger-scale solutions as well, including the BioRobot Universal and the clinically-targeted BioRobot MDx. Both platforms are designed for parallel processing of up to 96 samples, and combine Qiagen's proprietary membrane chemistry with a vacuum manifold and pipetting robots to automate the purification of DNA and RNA from a variety of sample types. These systems are also configured to prepare samples for PCR or reverse-transcriptase (RT)-PCR, although, as with the MagNA Pure LC, this still requires eventual manual transfer of reactions to a thermocycler instrument (Box 1). The Universal can also handle other complex nucleic-acid preparations, including the generation of microarray-ready samples from purified total RNA (Box 2). Although standardization is their primary focus, Qiagen also provides support for custom applications, such as RNA interference screens. "Molecular biologists from our service organization work directly with our customers, with the support of a worldwide network of application laboratories," says Leibinger.

\section{Special dispensation}

Brian Seed's team works with many automated laboratory systems, but he is unambiguous when asked which instruments have made the biggest strides. "Fluidhandling is at the top of the list for me, because it's so generic, and it's just about the most important thing in automation," he says. "It's gotten a whole lot better in terms of the process control interface, where you now have much more flexible tools to define the automation process." Indeed, recent years have seen the development of a new generation of small and relatively economically priced-but increasingly high-tech-systems for the general-purpose automation of biological procedures.

"The epMotion was generated exactly for that need, for all the people that have routine operations like serial dilutions, tube-to-plate transfers-all that nasty

\section{BOX 2 A CHIP OFF THE OLD `BOT}

In this 'post-genomic' era, much of the emphasis in nucleic-acid work has shifted from sequencing and cloning to RNA preparation for gene-expression analysis. This typically involves the use of microarrays, which in turn requires the precise and methodical-but also tedious and repetitive-preparation of many samples for hybridization. This need has not gone unnoticed among automation specialists; one company, Caliper Life Sciences, collaborated directly with microarray manufacturer Affymetrix to develop the Array Station, an instrument for the automated production of GeneChip microarrayready targets from previously purified RNA or DNA.

Beckman Coulter also worked with Affymetrix to develop a GeneChip-friendly targetpreparation method for their Biomek platforms. The resulting ArrayPLEX application can be run on the Biomek FX or 3000 in a 96-well format, with an automated process that requires only 18 hours to complete. "The customer puts total RNA and reagents on the deck," explains Roby, "and the system does the complementary DNA synthesis and clean-up, then the complementary RNA synthesis and clean-up, followed by fragmentation, quantitation and normalization. Following this, the samples are ready to be directly hybridized onto the chips." The ArrayPLEX application has met the performance standards to be designated an Affymetrix Premier Application late last year, and has also proven suitable for use with Agilent and even 'homebrew' microarrays.

Other manufacturers have thrown their hats into the ring as well-Qiagen has developed an Agilent- and Affymetrix-compatible sample preparation protocol for their BioRobot Universal system, and Hamilton Robotics offers a similar sample-preparation method for their MICROLAB STAR platform that also is compatible with Illumina's BeadChip format. Hamilton's Borner believes that the further refinement of such automated methods could be a major boon to the larger field of microarray research. "I think it's a really important technology that could become much more widespread if the costs were improved dramatatically, and if sample preparation could be faster and easier," he says. 
stuff you have to do every day," says Holger Eggert, a product manager at Eppendorf. Two models are currently available, both of which are capable of working with 96and 384-well plates as well as a variety of tube formats, and take advantage of Eppendorf's strengths as a pipette manufacturer for the precise manipulation of fluid volumes ranging from $1 \mu \mathrm{l}$ to $1 \mathrm{ml}$. 응 The primary difference between the two models is size: the 5075 features twelve rack positions, in comparison with the four incorporated into the 5070, and can also be integrated into a larger automated workflow. According to Eggert, the biggest advantage of the epMotion platforms is the ease with which they allow users to automate daily routines. "We have a setup and training time of less than a day," he says.

"The customer can set up whatever they like and use the tubes or plates they're used to... it's as flexible as possible, and easy to 을 go from manual to automated."

BioTek presently offers benchtop multiwell dispensing systems that operate at both

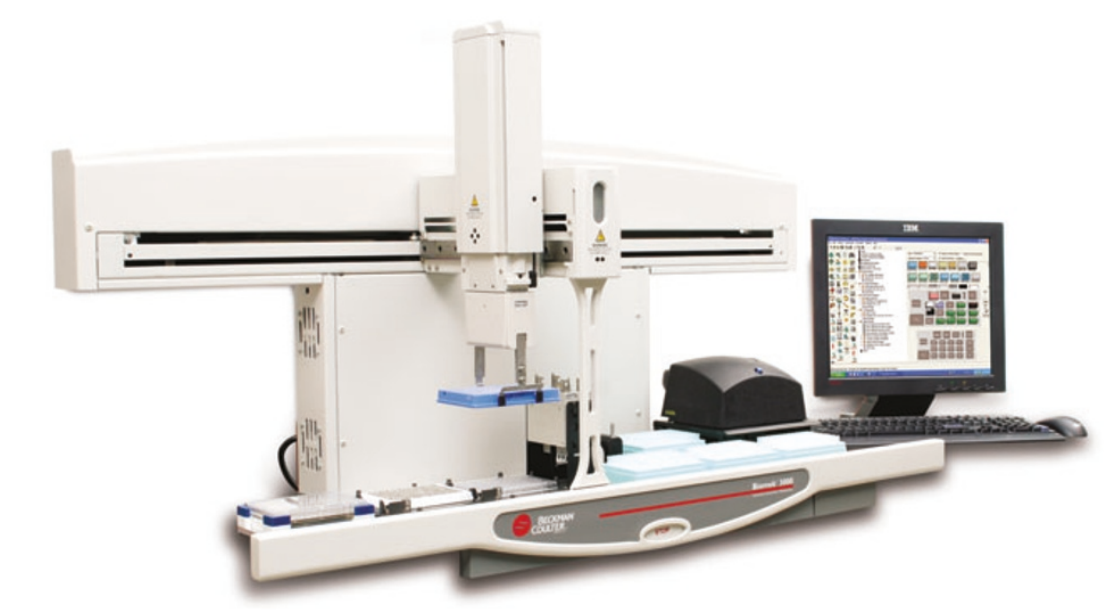

Beckman-Coulter offers a validated turnkey solution for automated target preparation for Affymetrix GeneChip experiments with their Biomek 3000-ArrayPLEX Workstation. (Courtesy of Beckman Coulter.)

extremes of the volume spectrum: between $5 \mu \mathrm{l}$ and $6 \mathrm{ml}$ for the $\mu$ Fill Microplate Dispenser, and between $100 \mathrm{nl}$ and $40 \mu \mathrm{l}$ for the newly launched NanoQuot unit. The $\mu$ Fill uses a microprocessor-controlled syringe pump that eliminates the need for recalibration, whereas NanoQuot uses a technology similar to that used in ink-jet printers to achieve accurate dispensing at low volumes. BioTek also manufactures the Precision series of personal pipetting robots, which provide a more sophisticated 
means for automating the multichannel pipetting of volumes between 1 and $200 \mu \mathrm{l}$, including a PC software interface that allows users to tweak virtually every aspect of the aspiration and dispensing process. According to product manager Jason Greene, systems like NanoQuot are primarily designed for people looking for a cost-effective means for automating very specific liquid-transfer tasks. In contrast, says Greene, "Customers who tend to see the Precision as most valuable are people who already have larger liquid-handling systems in their lab and are looking for something for smaller applications. I would consider it sort of a mid-throughput system."

\section{Welcome to the machine}

Although larger-scale liquid-handling platforms have generally not made their way into the average academic or clinical laboratory, such workstations are becoming more commonplace at university and hospital core facilities, giving researchers of even modest means and manpower the capability to considerably expand the throughput of their research.

Beckman Coulter recently launched two new models in its Biomek series of robotic platforms, the $\mathrm{FX}^{\mathrm{P}}$ and the $\mathrm{NX}^{\mathrm{P}}$, which expand the capabilities of the existing BioMek FX and NX units with enhancements for increased accuracy and performance in 96- and 384-well formats. The Biomek platforms are 'open' systems, compatible with a variety of third-party kits for assay automation, but Beckman
Coulter's 2005 acquisition of Agencourt Bioscience also means that the company is able to provide a variety of effective turnkey solutions for nucleic-acid preparation. The systems also benefit from an intuitive, icon-driven software interface. "Anybody, within half an hour or an hour of looking at how to create a method can create their own, and as they get more experienced, the methods can become more or less complex," explains applications manager Keith Roby. "You don't have to know Visual Basic or any kind of scripting-it's all drag-and-drop." Beckman Coulter also continues to release new applications, such as their recently developed Biomek 3000 GeXP Methods, which combine validated automation with Agencourt reagents to allow the rapid preparation of RNA for gene-expression assays.

Hamilton Life Science Robotics touts the versatility of its MICROLAB family of instruments, with the benchtop MICROLAB STARlet unit representing the smallest and most economical model. The STARlet is capable of working with both vacuum-based and magnetic bead-based nucleic acid preparation kits, and has the same capacity for customization as its larger siblings, allowing users to configure the number of pipetting channels, or incorporate a bar-code reader or colony picking unit. The STARlet also benefits from improved efficiency through enhancements to the pipetting process, such as Hamilton's compressed O-ring expansion (CO-RE) technology for tip attachment, which improves pick-up and ejection

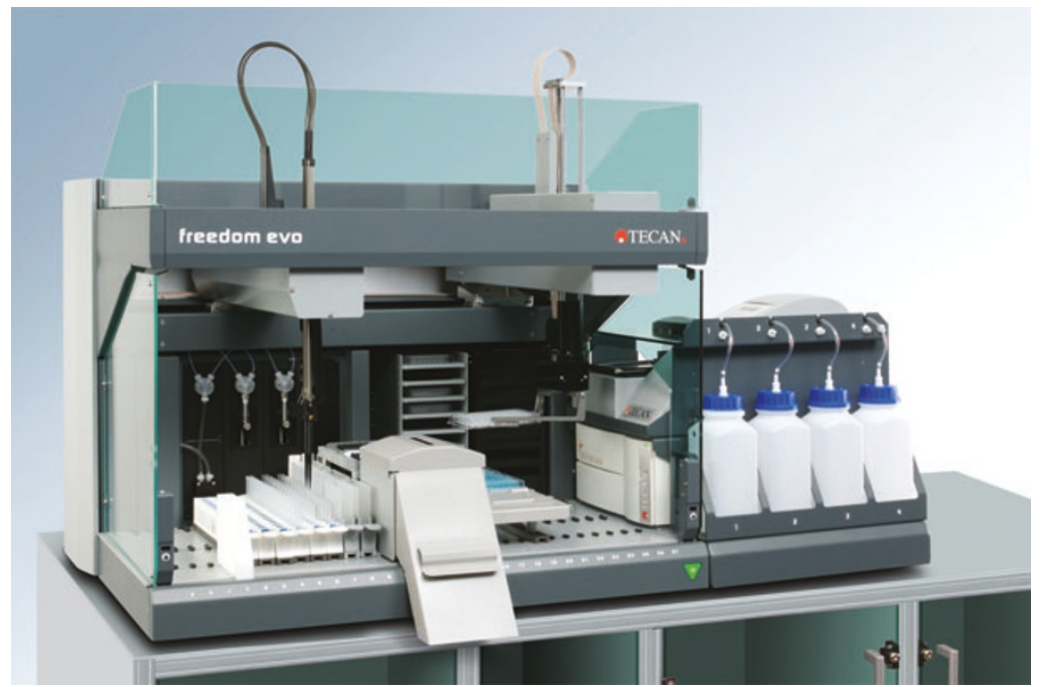

The Freedom EVO 75 represents the smallest and most economical of Tecan's versatile platforms for lab automation. (Courtesy of Tecan.) efficiency, reduces stress on the pipetting arm, and reduces aerosol production. The STARlet also continuously monitors fluid handling. "We have a pressure sensor inside our pipetting channels," explains Borner, "which tells us if the aspiration has been done properly or not."

Adaptibility is also a key asset of Tecan's Freedom EVO workstations, where users can customize their system by adding multiple robotic arms for applications that include microplate transport, multi channel pipetting for 96- or 384-well formats, or 'pick-and-place' arms for tube-handling and weigh-station applications. "It really takes a modular approach, so that the customer can build the instrument that they need," says Lauber. "There are probably over 30,000 variants that you can build with a Freedom EVO system." The incorporation of multiple arms also allows parallel processing of samples, and thus higher throughput. According to Lauber, Tecan is also developing more specialized, preconfigured systems for users with particular research applications in mind. Four different-sized units are available, but even the smallest unit, the Freedom EVO 75, offers the same range of adaptibility - and at a starting price of around $\$ 30,000$ for the basic unit, provides a reasonably costeffective entry into laboratory automation, with the potential for future expansion.

These systems offer impressive throughput for most users, but sometimes even academic researchers need more capacity. Researchers at the Genomics Institute of the Novartis Research Foundation (GNF) recently found themselves confronted with this problem when their lentiviral libraries finally grew beyond the capacity of their commercial robotic systems, forcing them to build a new solution. "GNF has an engineering department in-house, and they have recruited engineers from the car company Saturn," explains GNF researcher Sumit Chanda. "They brought engineering methods used to build cars into biology." The result was a fully automated platform for lentivirus-based genetic experiments. "We call it the ALVS, the automated lentiviral system, and it does everything: siRNA libraries, cDNA libraries and so on," explains Chanda. For research laboratories lacking automotive engineers, the ALVS is available through a newly launched company, GNF Systems, although the full system may prove too costly for individual labs. "It's really more cost effective for this to be set up at an 
institutional or departmental level, where you're going to set it all up and then perform ten or fifteen screens, with each lab banging away at the results," explains Anthony Orth, a colleague of Chanda. "We get 100 hits out of some of these screens, and each one is a paper waiting to happen.”

\section{Future upgrades}

Mechanization is definitely making its way into academia and the clinic, but this is clearly still an ongoing process. "I would really like to see automation become userfriendly enough that it could permeate the biological lab," says Seed. "But to really see this become effective, you'll need to have integrated solutions for commonly per- formed activities." Systems manufacturers also recognize this demand for stand-alone instruments that are flexible, yet simple enough to not require a dedicated technician. "They still want the full functionality," says Lauber, "but they want a smarter and easier-to-use automated system." To this end, many companies are focusing on their software, generating so-called 'wizards' that simplify the programming of instruments for multistage processes.

To Chanda's thinking, automation is an inevitable end of modern biology, even though cost remains a key obstacle. Prices have dropped for smaller instruments, with some basic liquid-handling units costing less than $\$ 15,000$, but this is still a major investment for many labs. Nonetheless, he believes that, as with microarrays, costs will soon fall to a point where robotics become a more practical - if not an essentialinvestment, and suggests that the launch of funding and development initiatives for genetics automation, similar to the US National Institutes of Health Roadmap program for high-throughput small-molecule screening, could be a huge asset in bringing such technology to academia more rapidly. "I think that most of the technology has been solved," he says, "It's just a question of access and exploitation.”

Michael Eisenstein is technology editor for Nature and Nature Methods. 


\section{SUPPLIHRS GUIDH: COMPANIHS OFHERING AUIOMANHD LABORAIORY SYSIFMS}

\section{Company}

Abbott Molecular

Abgene

Agilent

Applied Biosystems

Aurora Biomed

B-Bridge

Beckman Coulter

Biometra

BioTec

BioTek

Bruker Daltonics

CyBio

Deerac Fluidics

Eppendorf

Genetix

Genomic Solutions

Gentra

Gilson

Hamilton Robotics

KBiosystems

Labcyte

Lab Services BV

Microtec Nition

Millipore

Molecular Devices

Parallab
Bio-Rad Laboratories

Caliper Life Sciences

Corbett Life Sciences

Hudson Control Group

Magnetic Biosolutions

PerkinElmer

Promega

Protedyne

Qiagen

RoboDesign

Roche Applied Science

RTS Life Science

Sias

Tecan Group, Ltd.

The Automation Partnership

Thermo Electron

Titertek

Torcon Instruments

TTP Labtech

Velocity 11

Xiril

Zinsser Analytic

\section{Web address}

http://www.abbottmolecular.com

http://www.abgene.com

http://www.agilent.com

http://www.appliedbiosystems.com

http://www.aurorabiomed.com

http://www.b-bridge.com

http://www.bdbiosciences.com

http://www.beckman.com

http://www.biometra.de

http://www.bio-rad.com

http://www.biotec.co.jp

http://www.biotek.com

http://www.bdal.com

http://www.caliperls.com

http://www.corbettlifescience.com

http://www.cybio-ag.com

http://www.deerac.com

http://www.eppendorf.com

http://www.genetix.com

http://www.genomicsolutions.com

http://www.gentra.com

http://www.gilson.com

http://www.hamiltoncomp.com

http://www.hudsoncontrol.com

http://www.kbiosystems.com

http://www.labcyte.com

http://www.lab-services.nl

http://www.magbio.com

http://nition.com/en

http://www.millipore.com

http://www.moleculardevices.com

http://www.parallabs.com

http://las.perkinelmer.com

http://www.promega.com

http://www.protedyne.com

http://www.qiagen.com

http://www.robodesign.com

http://www.roche-applied-science.com

http://www.rtslifescience.com

http://www.sias.ch

http://www.tecan.com

http://www.automationpartnership.com

http://www.thermo.com

http://www.titertek.com

http://www.torconinstruments.com

http://www.ttplabtech.com

http://www.velocity11.com

http://www.xiril.com

http://www.zinsser-analytic.com 